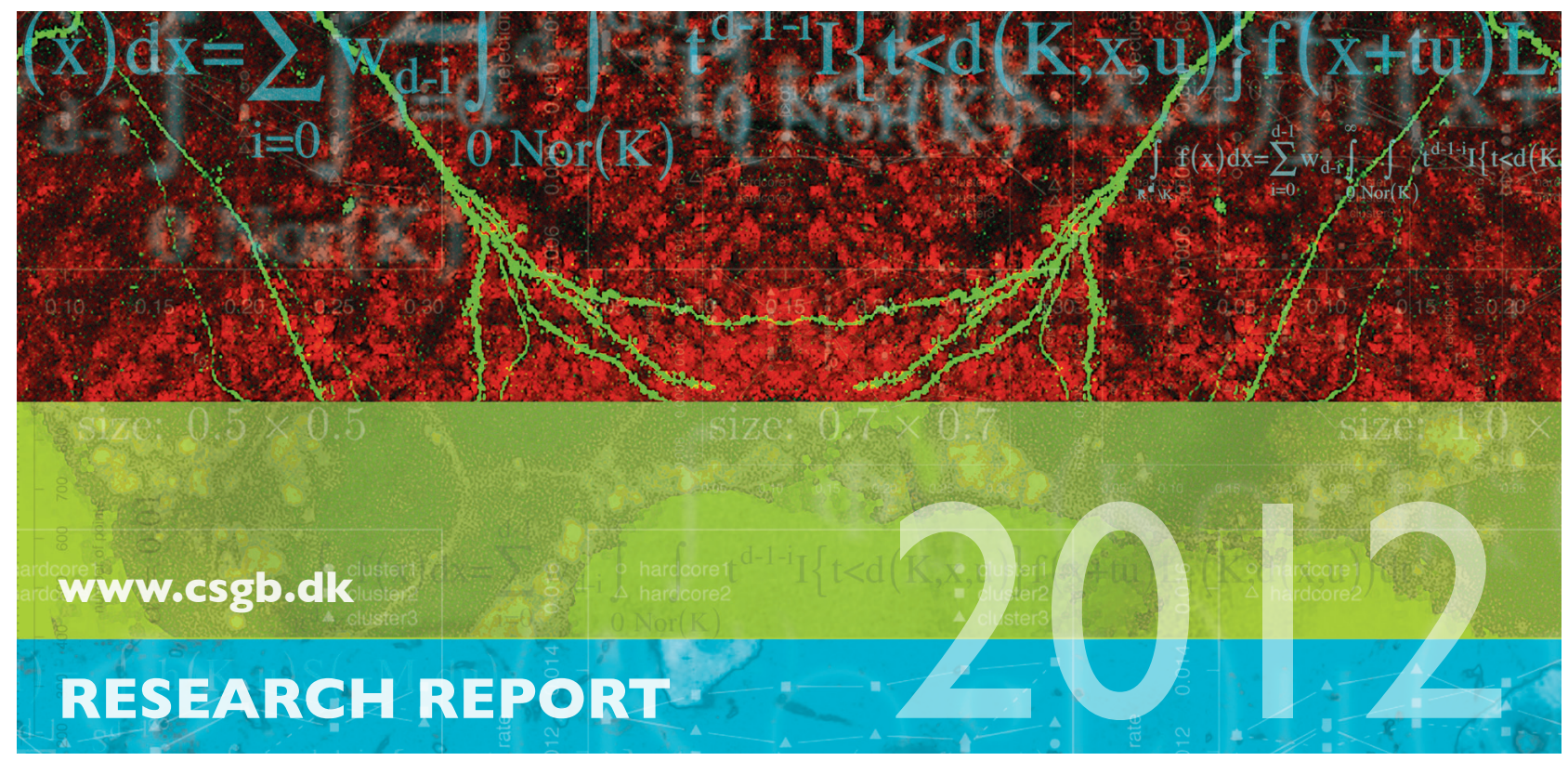

Linda V. Hansen and Thordis L. Thorarinsdottir

A note on moving average models for Gaussian random fields

No. 13, December 2012 


\title{
A note on moving average models for Gaussian random fields
}

\author{
Linda V. Hansen ${ }^{1}$ and Thordis L. Thorarinsdottir ${ }^{2}$ \\ ${ }^{1}$ Centre for Stochastic Geometry and Advanced Bioimaging, Aarhus University, Denmark \\ ${ }^{2}$ Norwegian Computing Center, Oslo, Norway
}

\begin{abstract}
The class of moving average models offers a flexible modeling framework for Gaussian random fields with many well known models such as the Matern covariance family and the Gaussian covariance falling under this framework. Moving average models may also be viewed as a kernel smoothing of a Lévy basis, a general modeling framework which includes several types of non-Gaussian models. We propose a new one-parameter spatial correlation model which arises from a power kernel and show that the associated Hausdorff dimension of the sample paths can take any value between 2 and 3 . As a result, the model offers similar flexibility in the fractal properties of the resulting field as the Matérn model.
\end{abstract}

Keywords: correlation function; Hausdorff dimension; moving average; power kernel; random field.

\section{Introduction}

In this note we consider isotropic Gaussian random fields that can be described as a kernel smoothing of a Gaussian measure. That is, let $X=\left\{X(s): s \in \mathbb{R}^{2}\right\}$ be the real valued random field given by

$$
X(s)=\int_{\mathbb{R}^{2}} k(\|s-u\|) L(\mathrm{~d} u)
$$

where $k$ is a deterministic kernel function which is a function of $u$ only via the Euclidean distance $\|s-u\|$ between $s$ and $u$, and $L$ is the centered, homogeneous Gaussian measure on the Borel subsets of $\mathbb{R}^{2}$. That is, $L$ is given by $L(\mathrm{~d} u) \sim$ $\mathcal{N}\left(0, \sigma^{2} \mathrm{~d} u\right)$, for $\sigma^{2}>0$, where $\mathrm{d} u$ denotes the Lebesgue measure on $\mathbb{R}^{2}$. The Gaussian distribution of the measure is carried over to the random field such that $X$ is again a Gaussian random field with mean zero and an isotropic covariance function $\operatorname{Cov}\left(X\left(s_{1}\right), X\left(s_{2}\right)\right)=\operatorname{Cov}\left(\left\|s_{1}-s_{2}\right\|\right)$. This model is typically known as a moving average model (Matérn, 1986; Yaglom, 1987; Oliver, 1995; Cressie and Pavlicová, 2002), and it is a special case of the linear spatio-temporal Lévy model proposed in 
Jónsdóttir et al. (2008). Hellmund et al. (2008) show that any stationary Gaussian random field with a continuous and integrable covariance function can be generated by a kernel smoothing of a homogeneous Gaussian measure.

More generally, the Gaussian measure in (1.1) may be replaced by a homogeneous Lévy basis. Multivariate stochastic processes that arise as a kernel smoothing of a Lévy basis have recently been considered in various modeling situations. Jónsdóttir et al. (2008) propose stochastic space-time growth models for planar objects where the stochastic term of the growth rate is modeled with such a process. Hellmund et al. (2008) discuss models for doubly stochastic point processes where the underlying spatial intensity measure is given by a kernel smoothing of a spatial Lévy basis, while Jónsdóttir et al. (2011) investigate this framework in an application to brain imaging. Processes on the sphere are studied in Hansen et al. (2011) in the context of modeling three dimensional star-shaped random sets and statistical inference for such models is discussed in Ziegel (2012).

The covariance structure of the random field $X$ determines the Hausdorff dimension of the graph $\operatorname{Gr} X=\left\{(t, X(t)): t \in \mathbb{R}^{2}\right\}$ which characterizes the roughness or the smoothness of the field. For a random field in $\mathbb{R}^{2}$, the Hausdorff dimension of the graph varies between 2 and 3, with the lower limit corresponding to a smooth, differentiable surface, while the upper limit corresponds to an excessively rough, space-filling surface (Falconer, 1990). The concept dates back to Hausdorff (1919) and the literature contains a rich discussion of Hausdorff dimension and the estimation thereof for Gaussian random fields over general Euclidean spaces, cf. Adler (2010), Xue and Xiao (2011), Gneiting et al. (2012), and references therein.

In Section 2, we investigate the fractal properties of the random field model in (1.1), as described by the Hausdorff dimension, for a number of parametric models for the kernel function $k$. Results for the Matérn covariance family, Gaussian covariances, and correlation functions arising from uniform kernels are briefly reviewed. For power kernels, we show that the modeling framework may yield fields associated with any Hausdorff dimension between 2 and 3 for negative powers, or a Hausdorff dimension of 2 for positive powers. A few inferential aspects of the power kernel model are listed in Section 3 and the note then closes with a discussion section.

\section{Fractal properties of moving average Gaussian random fields}

\subsection{Preliminaries}

Results of Jónsdóttir et al. (2008) imply that the random field $X$ in (1.1) has mean zero as the measure $L$ has zero mean. Similarly, $X$ has a constant variance $\operatorname{Var}(X(s))=2 \pi \sigma^{2} c_{2}$, where $c_{2}=\int_{0}^{\infty} r k(r)^{2} \mathrm{~d} r$. To obtain the covariance function, we use polar decomposition and Jónsdóttir et al. (2008, Eq. (11)) to find that

$$
\begin{aligned}
\operatorname{Cov} & \left(X\left(s_{1}\right), X\left(s_{2}\right)\right) \\
& =2 \sigma^{2} \int_{0}^{\infty} r k(r) \int_{0}^{\pi} k\left(\sqrt{r^{2}-2 r\left\|s_{1}-s_{2}\right\| \cos \varphi+\left\|s_{1}-s_{2}\right\|^{2}}\right) \mathrm{d} \varphi \mathrm{d} r .
\end{aligned}
$$


In general, the random field is stationary if the covariance function depends on the spatial separation vector $s_{1}-s_{2}$ only and it is isotropic if the covariance function depends on the distance $\left\|s_{1}-s_{2}\right\|$ only, which is the case in (2.1). Furthermore, the correlation function of $X$ becomes

$$
C(h)=\frac{1}{\pi c_{2}} \int_{0}^{\infty} r k(r) \int_{0}^{\pi} k\left(\sqrt{r^{2}+h^{2}-2 r h \cos \varphi}\right) \mathrm{d} \varphi \mathrm{d} r .
$$

Assume, for some $\alpha$, that

$$
\begin{aligned}
\alpha & =\sup \left\{\beta>0:[C(0)-C(h)]=o\left(h^{\beta}\right), h \downarrow 0\right\} \\
& =\inf \left\{\beta>0: h^{\beta}=o([C(0)-C(h)]), h \downarrow 0\right\}
\end{aligned}
$$

Then, with probability one, the Hausdorff dimension of the graph $\operatorname{Gr} X$ is $D(\operatorname{Gr} X)=$ $3-\alpha / 2$, where $\alpha$ is called the fractal index of $X$ (Adler, 2010). If, in the equation above, the supremum is smaller than the infimum, then the Hausdorff dimension of the graph will be determined by the upper bound inf $\left\{\beta>0: h^{\beta}=\right.$ $o([C(0)-C(h)]), h \downarrow 0\}$, see Theorem 3.8 in Xiao (2007).

In the following, we study the behavior of $[C(0)-C(h)]$ for a number of parametric models for the isotropic kernel function $k$ in (1.1). An overview over the models discussed below is given in Table 1.

\subsection{Models of varying Hausdorff dimension}

The model in (1.1) may yield the Matérn covariance family (Matérn, 1986; Guttorp and Gneiting, 2006) with correlation function

$$
C(h)=\frac{(\lambda h)^{\nu}}{2^{\nu-1} \Gamma(\nu)} K_{\nu}(\lambda h)
$$

where $\lambda>0$ is a spatial scale parameter, $\nu>0$ is a smoothness parameter, $\Gamma(\cdot)$ denotes the gamma function, and $K_{\nu}(\cdot)$ is the modified Bessel function of the second kind. The Matérn family is closed under convolution in that the correlation functions above results from (1.1) by applying the kernel function

$$
k(r)=\frac{\lambda^{\frac{\nu+3}{2}}}{\pi 2^{\frac{\nu+1}{2}} \Gamma\left(\frac{\nu+1}{2}\right)} r^{\frac{\nu-1}{2}} K_{\frac{\nu-1}{2}}(\lambda r)
$$

(Matérn, 1986; Gneiting et al., 2010; Jónsdóttir et al., 2011). The smoothness parameter $\nu$ defines the Hausdorff dimension of the graph $\operatorname{Gr} X$ which equals the maximum of 2 and $3-\nu$, see e.g. Adler (2010) and Goff and Jordan (1988).

Hansen et al. (2011) prove that the surface of a three dimensional particle which radial function is given by a kernel smoothing of a Gaussian measure with a power kernel will obtain any desired Hausdorff dimension. In the planar case, this power kernel is given by

$$
k(r)= \begin{cases}r^{-q}-1, & 0<r \leq 1 \\ 0, & \text { otherwise }\end{cases}
$$


for $0<q<1$. The associated correlation function (2.2) takes the form

$$
C(h)=\frac{1}{\pi c_{2}} \int_{0}^{1} r\left[r^{-q}-1\right] \int_{A_{h}(r)}\left[g(h, r, \varphi)^{-q}-1\right] \mathrm{d} \varphi \mathrm{d} r,
$$

where $g(h, r, \varphi)=\sqrt{r^{2}+h^{2}-2 r h \cos \varphi}$ and $A_{h}(r)=\{\varphi \in[0, \pi]: g(h, r, \varphi) \in$ $(0,1]\}$. While the correlation function is not available in closed form, it can be shown that the Hausdorff dimension of $\operatorname{Gr} X$ is equal to $2+q$, see Theorem 2.1 below. The parameter $q$ is thus a smoothness parameter which here defines the fractal properties of the resulting sample paths similar to the parameter $\nu$ in the Matérn model above.

Theorem 2.1. Let $C$ be the correlation function in (2.2) with $k$ the power kernel in (2.3) for $0<q<1$. Then it holds that

$$
[C(0)-C(h)] \sim a_{q} h^{2(1-q)}, \quad h \rightarrow 0,
$$

where

$$
a_{q}=\frac{\pi^{2} q^{2}}{2(1-q)^{3}(2-q)} \frac{\Gamma\left(1-\frac{1}{2} q\right)^{2} \Gamma(q)}{\Gamma\left(\frac{1}{1} q\right)^{2} \Gamma(1-q)} .
$$

Proof. Let $0 \leq h \leq 1$ and $0<r<1$. Then,

$$
\frac{C(0)-C(h)}{\pi c_{2}}=\int_{0}^{1} r\left[r^{-q}-1\right]\left\{\int_{0}^{\pi}\left[r^{-q}-1\right] \mathrm{d} \varphi-\int_{A_{h}(r)}\left[g(h, r, \varphi)^{-q}-1\right] \mathrm{d} \varphi\right\} \mathrm{d} r .
$$

For $0<r<1-h$ it holds that $A_{h}(r)=[0, \pi]$, whereas $A_{h}(r) \subset[0, \pi]$, for $1-h<$ $r<1$. Hence, we conduct the integration with respect to $r$ in a two-step procedure. First, note that a Taylor expansion around zero implies that $\left((1-h)^{-q}-1\right)=\mathcal{O}(h)$. Furthermore, for $h$ small enough, say $h<h_{0}$, the integral difference within the curly brackets is smaller than one. Now, for $h<h_{0}$, an application of the First Mean Value Theorem for Integration yields that there exists $t \in(1-h, 1)$ such that

$$
\begin{array}{rl}
\int_{1-h}^{1} & r \\
& {\left[r^{-q}-1\right]\left\{\int_{0}^{\pi}\left[r^{-q}-1\right] \mathrm{d} \varphi-\int_{A_{h}(r)}\left[g(h, r, \varphi)^{-q}-1\right] \mathrm{d} \varphi\right\} \mathrm{d} r} \\
& =h t\left[t^{-q}-1\right]\left\{\int_{0}^{\pi}\left[t^{-q}-1\right] \mathrm{d} \varphi-\int_{A(t)}\left[g(h, t, \varphi)^{-q}-1\right] \mathrm{d} \varphi\right\} \\
& \leq h\left[(1-h)^{-q}-1\right] \\
& =\mathcal{O}\left(h^{2}\right) .
\end{array}
$$

For the remaining integral term, applying the substitution $r=h x$ yields

$$
\begin{array}{rl}
\int_{0}^{1-h} & r\left[r^{-q}-1\right] \int_{0}^{\pi}\left[r^{-q}-g(h, r, \varphi)^{-q}\right] \mathrm{d} \varphi \mathrm{d} r \\
& =h^{2(1-q)} \int_{0}^{\frac{1-h}{h}} x\left[x^{-q}-h^{q}\right] \int_{0}^{\pi}\left[x^{-q}-g(1, x, \varphi)^{-q}\right] \mathrm{d} \varphi \mathrm{d} x .
\end{array}
$$


For $x \rightarrow 0$ we obtain

$$
\begin{aligned}
\int_{0}^{\pi}\left[x^{-q}-g(1, x, \varphi)^{-q}\right] \mathrm{d} \varphi \\
\quad=\pi x^{-q}-\int_{0}^{\pi} g(1, x, \varphi)^{-q} \mathrm{~d} \varphi=\pi x^{-q}+\mathcal{O}\left(x^{0}\right) \sim \pi x^{-q} .
\end{aligned}
$$

Furthermore, for $x \rightarrow \infty$ a Taylor expansion of $(1+y)^{-q / 2}$ around $y=0$ yields

$$
\int_{0}^{\pi}\left[x^{-q}-g(1, x, \varphi)^{-q}\right] \mathrm{d} \varphi=x^{-q} \int_{0}^{\pi}\left[1-g\left(1, x^{-1}, \varphi\right)^{-q}\right] \mathrm{d} \varphi \sim-\frac{\pi q^{2}}{4} x^{-q-2} .
$$

Hence, we get the following limit result

$$
\begin{gathered}
\int_{0}^{\frac{1-h}{h}} x\left[x^{-q}-h^{q}\right] \int_{0}^{\pi}\left[x^{-q}-g(1, x, \varphi)^{-q}\right] \mathrm{d} \varphi \mathrm{d} x \\
\underset{h \rightarrow 0}{\longrightarrow} \int_{0}^{\infty} x^{1-q} \int_{0}^{\pi}\left[x^{-q}-g(1, x, \varphi)^{-q}\right] \mathrm{d} \varphi \mathrm{d} x .
\end{gathered}
$$

It follows from Hansen et al. (2012, Thm. 2) that

$$
\int_{0}^{\infty} x^{1-q} \int_{0}^{\pi}\left[x^{-q}-g(1, x, \varphi)^{-q}\right] \mathrm{d} \varphi \mathrm{d} x=\frac{\pi}{2(1-q)^{2}} \frac{\Gamma\left(1-\frac{1}{2} q\right)^{2} \Gamma(q)}{\Gamma\left(\frac{1}{1} q\right)^{2} \Gamma(1-q)} .
$$

This result, together with

$$
c_{2}=\int_{0}^{1} r\left[r^{-q}-1\right]^{2} \mathrm{~d} r=\frac{q^{2}}{2(2-q)(1-q)},
$$

concludes the proof.

\subsection{Models of fixed Hausdorff dimension}

The power kernel in (2.3) may also be considered for positive powers. That is, let

$$
k(r)= \begin{cases}1-r^{q}, & 0<r \leq 1 \\ 0, & \text { otherwise }\end{cases}
$$

for $q>0$. The associated correlation function (2.2) is here given by

$$
C(h)=\frac{1}{\pi c_{2}} \int_{0}^{1} r\left[1-r^{q}\right] \int_{A_{h}(r)}\left[1-g(h, r, \varphi)^{q}\right] \mathrm{d} \varphi \mathrm{d} r,
$$

with $g(h, r, \varphi)$ and $A_{h}(r)$ defined as above. To calculate the fractal index of $X$ under this model, similar arguments as in the proof of Theorem 2.1 show that

$$
\frac{q^{2} \pi}{2(q+1)(q+2)}[C(0)-C(h)] \sim \mathcal{O}\left(h^{2}\right)+\int_{0}^{1-h} r\left[1-r^{q}\right] \int_{0}^{\pi}\left[g(h, r, \varphi)^{q}-r^{q}\right] \mathrm{d} \varphi \mathrm{d} r .
$$


For the remaining integral, the First Mean Value Theorem for Integration yields that

$$
\int_{0}^{1-h} r\left[1-r^{q}\right] \int_{0}^{\pi}\left[g(h, r, \varphi)^{q}-r^{q}\right] \mathrm{d} \varphi \mathrm{d} r \leq(1-h)^{2}\left|\int_{0}^{\pi}\left[g(h, r, \varphi)^{q}-r^{q}\right] \mathrm{d} \varphi\right| .
$$

Further, a Taylor approximation of $f(h)=g(h, r, \varphi)^{q}-r^{q}$ around $h=0$ shows that

$$
\int_{0}^{\pi}\left[g(h, r, \varphi)^{q}-r^{q}\right] \mathrm{d} \varphi=\mathcal{O}\left(h^{2}\right)
$$

The graph $\operatorname{Gr} X$ for the resulting random field $X$ thus has Hausdorff dimension 2 independent of the value of $q$.

A smooth, differentiable surface may also be obtained with a Gaussian kernel,

$$
k(r)=\frac{1}{2 \pi \omega^{2}} \exp \left(-\frac{r^{2}}{2 \omega^{2}}\right)
$$

with mean zero and variance $\omega^{2}$. The correlation function is here given by $C(h)=$ $\exp \left(-h^{2} /\left(4 \omega^{2}\right)\right)$, see e.g. Matérn (1986), from which it can easily be seen that $D(\operatorname{Gr} X)=2$ as for the power kernel in (2.4) above.

Both Jónsdóttir et al. (2011) and Hansen et al. (2011) consider a bounded uniform kernel which here takes the form $k(r)=\mathbb{1}\{r \leq R\} /\left(\pi R^{2}\right)$ for $R>0$, where $\mathbb{1}\{\cdot\}$ denotes the indicator function. As shown e.g. in Jónsdóttir et al. (2011), the correlation function (2.2) becomes

$$
C(h)=\left[\frac{2}{\pi} \arccos \left(\frac{h}{2 R}\right)-\frac{1}{2 \pi h R^{2}} \sqrt{4 R^{2}-h^{2}}\right] \mathbb{1}\{h \leq 2 R\}
$$

from which it directly follows that $D(\operatorname{Gr} X)=2.5$. This result coinsides with the corresponding result on the sphere (Hansen et al., 2011). 
Table 1: The kernel functions $k(r)$ discussed in this note, the respective parameters, the resulting correlation functions $C(h)$, and the associated Hausdorff dimension of the sample paths $D(\operatorname{Gr} X)$.

\begin{tabular}{cccc}
\hline & Parameter & $C(h)$ & $D(\operatorname{Gr} X)$ \\
\hline$\frac{\lambda^{\frac{\nu+3}{2}} r^{\frac{\nu-1}{2}}}{\pi 2^{\frac{\nu+1}{2}} \Gamma\left(\frac{\nu+1}{2}\right)} K_{\frac{\nu-1}{2}}(\lambda r)$ & $\lambda>0, \nu>0$ & $\frac{(\lambda h)^{\nu}}{2^{\nu-1} \Gamma(\nu)} K_{\nu}(\lambda h)$ & $\max \{2,3-\nu\}$ \\
$\mathbb{1}\{r \in(0,1]\}\left[r^{-q}-1\right]$ & $q \in(0,1)$ & $\frac{1}{\pi c_{2}} \int r k(r) \int_{A_{h}(r)}\left[g(h, r, \varphi)^{-q}-1\right] \mathrm{d} \varphi \mathrm{d} r$ & $2+q$ \\
$\mathbb{1}\{r \leq R\} /\left[\pi R^{2}\right]$ & $R \geq 0$ & $\mathbb{1}\{h \leq 2 R\}\left[\frac{2}{\pi} \arccos \left(\frac{h}{2 R}\right)-\frac{\sqrt{4 R^{2}-h^{2}}}{2 \pi h R^{2}}\right]$ & 2.5 \\
$\mathbb{1}\{r \in(0,1]\}\left[1-r^{q}\right]$ & $q>0$ & $\frac{1}{\pi c_{2}} \int r k(r) \int_{A_{h}(r)}\left[1-g(h, r, \varphi)^{q}\right] \mathrm{d} \varphi \mathrm{d} r$ & 2 \\
$\frac{1}{2 \pi \omega^{2}} \exp \left(-\frac{r^{2}}{2 \omega^{2}}\right)$ & $\omega \geq 0$ & $\exp \left(-\frac{h^{2}}{4 \omega^{2}}\right)$ & 2 \\
\hline
\end{tabular}

\section{Inferential aspects of the power kernel model}

Moving average models based on the power kernels in (2.3) and (2.4) are simple in the sense that they only have two parameters, the smoothness parameter $q$ and the variance of the underlying measure $\sigma^{2}$. However, inference methods such as maximum likelihood inference are somewhat complicated by the lack of a closed form for the correlation function. An approximative inference for both the kernel and the underlying measure could be provided by the general Bayesian inference framework described in Wolpert and Ickstadt (1998). The advantage of this framework is its flexibility, the method does not rely on the Gaussian assumption, while its downside is the computational complexity. For the negative power kernel, there is a direct link between the fractal properties of the field and the smoothness parameter $q$. In this case, $q$ can be estimated by applying any of the methods for estimating the fractal dimension of spatial data described in Gneiting et al. (2012). The variance parameter $\sigma^{2}$ may then be estimated e.g. by applying the method of moments.

When the data is observed on a lattice, inference is often performed using the spectral representation of the data, see e.g. Fuentes and Reich (2010) and references therein. The spectral density of the random field $X$ in (1.1) under the power kernel in (2.3) is given by

$$
f(\omega)=\frac{\pi \sigma^{2}}{4} \frac{q^{2}}{(2-q)(1-q)}\left(\sum_{n=0}^{+\infty}(-1)^{n}\left(\frac{1}{(n+1-q / 2)}-\frac{1}{n+1}\right) \frac{\|\omega\|^{2 n}}{2^{2 n} n ! \Gamma(n+1)}\right)^{2} .
$$

To see this, recall the definition of the spectral density under an isotropic moving average model

$$
f(\omega)=\frac{1}{(2 \pi)^{2}} \int_{\mathbb{R}^{2}} \operatorname{Cov}(\|s\|) e^{-i \omega \cdot s} \mathrm{~d} s=\frac{1}{(2 \pi)^{2}} \operatorname{Var}(X)\left[\int_{\mathbb{R}^{2}} k(\|s\|) e^{-i \omega \cdot s} \mathrm{~d} s\right]^{2},
$$


where $\operatorname{Cov}(\|s\|)=\operatorname{Var}(X) \int_{\mathbb{R}^{2}} k(\|u\|) k(\|s+u\|) \mathrm{d} u$. Polar decomposition, formula 10.32.1 of Digital Library of Mathematical Functions (2011), and Wolfram Function Site (2001), respectively, yield

$$
\begin{aligned}
\int_{\mathbb{R}^{2}} k(\|s\|) e^{-i \omega \cdot s} \mathrm{~d} s & =\int_{0}^{1} r k(r) \int_{0}^{2 \pi} \exp (-i r\|\omega\| \cos \theta) \mathrm{d} \theta \mathrm{d} r \\
& =2 \pi \int_{0}^{1} r\left(r^{-q}-1\right) J_{0}(r\|\omega\|) \mathrm{d} r \\
& =\frac{2 \pi}{2-q}{ }_{1} F_{2}\left(1-\frac{q}{2} ; 1,2-\frac{q}{2} ;-\frac{\|\omega\|^{2}}{4}\right)-2 \pi\|\omega\|^{-1} J_{1}(\|\omega\|),
\end{aligned}
$$

where $J_{0}$ and $J_{1}$ are Bessel functions and ${ }_{1} F_{2}$ is a generalized hypergeometric function.

For the positive power kernel in (2.4), the spectral density becomes

$$
f(\omega)=\frac{\pi \sigma^{2} q^{2}}{(2+q)(1+q)}\left[\|\omega\|^{-1} J_{1}(\|\omega\|)-\frac{1}{2+q}{ }_{1} F_{2}\left(1+\frac{q}{2} ; 1,2+\frac{q}{2} ;-\frac{\|\omega\|^{2}}{4}\right)\right]^{2} .
$$

When $q=2$, the density in (3.1) equals $f(\omega)=4 \pi \sigma^{2}\|\omega\|^{-4} J_{2}(\|\omega\|)^{2} / 3$. As $J_{2}(r)^{2}=$ $\mathcal{O}\left(r^{-1}\right)$ when $r \rightarrow \infty$ (Digital Library of Mathematical Functions, 2011, formula 10.7.8) it follows that the sample paths of $X$ are differentiable for $q=2$, see Section 1.4.1. in Adler (2010) and Theorem 3.2 in Potthoff (2010), or Corollary 5.3.17 in Scheuerer (2009).

\section{Discussion}

Kernel smoothing of a Lévy basis offers a general and flexible framework for multivariate stochastic processes. We consider a special case, moving average Gaussian random fields, and show that smoothing of a power kernel with a Gaussian measure results in fields whose graph can attain any Hausdorff dimension between 2 and 3 for negative powers and a Hausdorff dimension equal to 2 for positive powers. Even though the correlation function is not available in closed from, this one-parameter correlation model offers an appealing alternative to the Matérn family which also provides fields with varying associated Hausdorff dimension.

We have here focused on isotropic covariance models. Isotropy, along with stationarity, are clearly not always realistic assumptions. Under the Gaussian assumption, the correlation function depends only on the associated kernel function. A natural extension of the current framework to non-isotropic or non-stationary covariance models thus involves applying non-isotropic or spatially varying kernel functions. Extensions in this direction for similar models are discussed in Higdon et al. (1999) and Paciorek and Schervish (2006), where the Euklidean distance between location is replaced by a spatially varying Mahalanobis distance, and in Vianna Neto et al. (2012), where non-stationary is obtained through spatially varying covariates. The power kernel model is especially appealing for such extensions in that the kernel function itself is a very simple function and it is thus straightforward to obtain the desired variability in the kernel. 
Similarly, the Gaussian assumption can easily be relaxed by considering nonGaussian Lévy basis, see Jónsdóttir et al. (2008). An example where a gamma basis is utilized can be found in Wolpert and Ickstadt (1998). While non-Gaussian theory on the Hausdorff dimension remains elusive, the results of Hansen et al. (2011) indicate that the smoothness of the surface of a Gamma random field is likely to correspond to that of a Gaussian random field with the same correlation structure. Shieh and Xiao (2010) provide results for the Hausdorff dimension of the sample paths of self-similar stable random fields which might provide insight in this case. However, the random fields obtained using the kernel functions in Table 1 with a non-Gaussian Lévy basis are generally not self-similar. Results regarding regularity of the sample paths for non-Gaussian random fields are given in Scheuerer (2010).

\section{Acknowledgements}

The authors thank Tilmann Gneiting, Eva B. Vedel Jensen, Jens Ledet Jensen, Kristjana Ýr Jónsdóttir, and Michael Scheuerer for comments and discussions. This research has been supported by the Centre for Stochastic Geometry and Advanced Bioimaging at Aarhus University which is funded by a grant from the Villum Foundation, by the German Research Foundation (DFG) within the program "Spatio/Temporal Graphical Models and Applications in Image Analysis", grant GRK 1653, and by the $\mathrm{SFI}^{2}$, Statistics for Innovation, Oslo.

\section{References}

Robert J. Adler. The Geometry of Random Fields. SIAM, Philadelphia, SIAM Classics edition, 2010.

N. Cressie and M. Pavlicová. Calibrated spatial moving average simulations. Statistical Modelling, 2:267-279, 2002.

Digital Library of Mathematical Functions, 2011. Release 2011-07-01, http://dlmf .nist. gov.

Kenneth Falconer. Fractal Geometry. John Wiley and Sons, Chichester, 1990.

M Fuentes and B Reich. Spectral domain. In A. E. Gelfand, P. J. Diggle, M. Fuentes, and P. Guttorp, editors, Handbook of Spatial Statistics, pages 57-78. Chapman and Hall/CRC: Boca Raton, 2010.

T. Gneiting, W. Kleiber, and M. Schlather. Matérn cross-covariance functions for multivariate random fields. Journal of the American Statistical Association, 105:1167-1177, 2010.

T. Gneiting, H. Ševčiková, and D. B. Percival. Estimators of fractal dimension: Assessing the roughness of time series and spatial data. Statistical Science, 27:247-277, 2012.

J. A. Goff and T. H. Jordan. Stochastic modeling of seafloor morphology: Inversion of sea beam data for second-order statistics. Journal of Geophysical Research, 93:13589-13608, 1988. 
P. Guttorp and T. Gneiting. Studies of the history of probability and statistics XLIX: On the Matérn correlation family. Biometrika, 93:989-995, 2006.

L. V. Hansen, T. L. Thorarinsdottir, and T. Gneiting. Lévy particles: Modelling and simulating star-shaped random sets. Technical Report No. 04, September 2011, Centre for Stochastic Geometry and Advanced Bioimaging, 2011. http://data.imf.au.dk/ publications/csgb/2011/imf-csgb-2011-04.pdf.

L. V. Hansen, T. L. Thorarinsdottir, T. Gneiting, and D. Richards. Lévy particles: Modelling and simulating star-shaped random sets. In submission, 2012.

Felix Hausdorff. Dimension und äußeres Maß. Mathematische Annalen, 79:157-179, 1919.

Gunnar Hellmund, M. Prokešová, and Eva B. Vedel Jensen. Lévy based Cox point processes. Advances in Applied Probability, 40:603-629, 2008.

D. Higdon, J. Swall, and J. Kern. Non-stationary spatial modeling. In J. Bernardo, J. Berger, A. Dawid, and A. Smith, editors, Bayesian Statistics 6, pages 761-768. Oxford University Press, Oxford, UK, 1999.

K. Y. Jónsdóttir, A. Rønn-Nielsen, K. Mouridsen, and E. B. V. Jensen. Lévy based modelling in brain imaging. Technical Report No. 02, April 2011, Centre for Stochastic Geometry and Advanced Bioimaging, 2011. http://data.imf.au.dk/publications/ csgb/2011/imf-csgb-2011-02.pdf.

Kristjana Yr Jónsdóttir, Jßrgen Schmiegel, and Eva B. Vedel Jensen. Lévy based growth models. Bernoulli, 14:62-90, 2008.

B. Matérn. Spatial Variation. Berlin: Springer-Verlag, 2nd edition, 1986.

D. S. Oliver. Moving averages for Gaussian simulation in two and three dimensions. Mathematical Geology, 27:939-960, 1995.

C. J. Paciorek and M. J. Schervish. Spatial modelling using a new class of nonstationary covariance functions. Environmetrics, 17:483-506, 2006.

J. Potthoff. Sample properties of random fields II: Differentiability. Communications in Stochastic Analysis, 4:335-353, 2010.

M. Scheuerer. A comparison of models and methods for spatial interpolation in statistics and numerical analysis. PhD thesis, Georg-August-Universität Göttingen, 2009. Available at http://webdoc.sub.gwdg.de/diss/2010/scheuerer/.

M. Scheuerer. Regularity of the sample paths of a general second order random field. Stochastic Processes and their Applications, 120:1879-1897, 2010.

Narn-Rueih Shieh and Yimin Xiao. Hausdorff and packing dimension of the images of random fields. Bernoulli, 16(4):926-952, 2010.

J. H. Vianna Neto, A. M Schmidt, and P Guttorp. Accounting for spatially varying directional effects in spatial covariance structures. arXiv:1209.5977 [stat.AP], 2012.

Wolfram Function Site, 2001. Modification date 2001-10-29, http://functions . wolfram. com/03.01.21.0007.01. 
Robert L. Wolpert and Katja Ickstadt. Poisson/gamma random field models for spatial statistics. Biometrika, 85:251-267, 1998.

Y. Xiao. Strong local nondeterminism and sample path properties of Gaussian random fields. In T. L. Lai, Q. Shao, and L. Qian, editors, Asymptotic Theory in Probability and Statistics with Applications, pages 136-176. Higher Education Press: Beijing, 2007.

Y. Xue and Y. Xiao. Fractal and smoothness properties of space-time Gaussian models. Frontiers of Mathematics in China, 6:1217-1248, 2011.

A. M. Yaglom. Correlation theory of stationary and related random functions. Volume I: Basic results. Springer, Berlin, 1987.

J. Ziegel. Stereological modelling of random particles. Communications in Statistics Theory and Methods, page to appear, 2012. 